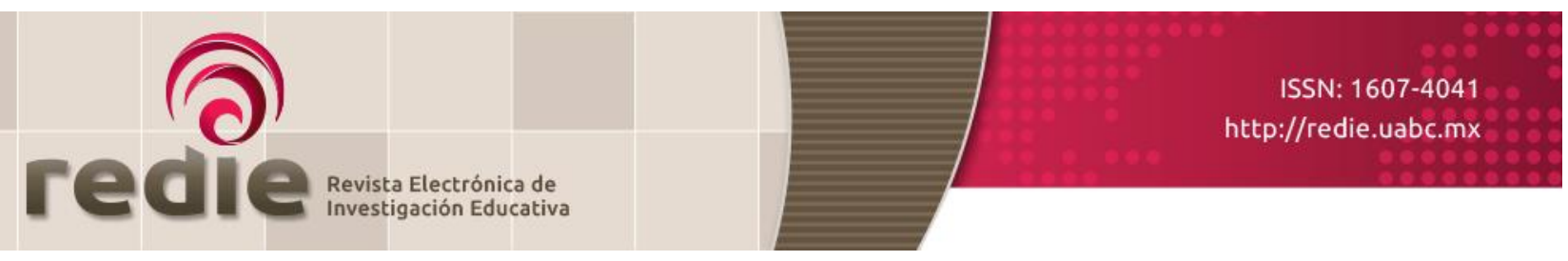

Vol. 21, 2019/e03

\title{
Evaluación comunicativa y selección de contenidos en contextos escolares vulnerables chilenos
}

\section{Communicative Evaluation and Content Selection in Vulnerable School Contexts in Chile}

\author{
Miguel Del Pino-Sepúlveda (*) mdelpino@uct.cl \\ Elizabeth Montanares-Vargas (*) emontanares@uct.cl \\ (*) Universidad Católica de Temuco \\ (Recibido: 18 de mayo de 2017; Aceptado para su publicación: 23 de junio de 2017)
}

Cómo citar: Del Pino-Sepúlveda, M. y Montanares-Vargas, E. (2019). Evaluación comunicativa y selección de contenidos en contextos escolares vulnerables chilenos. Revista Electrónica de Investigación Educativa, 21, e03, 1-12. doi:10.24320/redie.2019.21.e03.1984

\begin{abstract}
Resumen
El trabajo aborda la evaluación de contenidos desde el enfoque de la evaluación comunicativa y tiene tres objetivos: 1) Develar los contenidos que se trabajan en evaluación comunicativa desde la perspectiva de los agentes educativos involucrados, 2) Identificar el rol de los participantes en la selección de contenidos a evaluar, y 3) Identificar los criterios de selección de contenidos. Se empleó la metodología DialógicaKishu Kimkelay Ta Che, y como técnicas de recogida de información la conversación dialógica y el diálogo colectivo en tres ciudades: Concepción, Tomé y Talca (Chile). La validación de los datos se realizó a través de la categorización de los mismos en dimensiones transformadoras, conservadoras y exclusoras. Como resultados relevantes se propone incorporar y rescatar saberes sociales, culturales y lingüísticos de las comunidades a evaluar con el fin de enriquecer los contenidos prescritos por el currículum nacional.
\end{abstract}

Palabras clave: Evaluación, evaluación de la educación, evaluación del currículum, contenido del curso.

\section{Abstract}

This work addresses content evaluation from a communicative evaluation approach and has three objectives: 1) Uncover the content of communicative evaluation from the perspective of education actors involved, 2) Identify the role of participants in selecting content to be evaluated, and 3) Identify content selection criteria. The Dialogic-Kishu Kimkelay Ta Che methodology was used, and dialogic conversation and collective dialogue were used to collect information in three cities: Concepción, Tomé, and Talca (Chile). To validate the data, it was categorized into transformative, conservative, and exclusionary dimensions. Relevant results suggest integrating and salvaging the social, cultural, and linguistic knowledge of communities evaluated, with the goal of enriching the content prescribed by the national curriculum. 


\section{Introducción}

La evaluación en Chile es un tema que provoca controversias por los numerosos mecanismos reguladores de la calidad de los aprendizajes; destacan entre ellos el Sistema de Medición de la Calidad en Educación (SIMCE), la Prueba Selección Universitaria (PSU), la evaluación de formación docente (Evaluación Diagnóstica Nacional, antigua Prueba Inicia) y la evaluación de docentes en ejercicio (Evaluación Docente), dispositivos de regulación y control que intentan dar respuesta al paradigma de Efectividad y Mejora Escolar (Ferrada, 2012).

En el caso del SIMCE -que evalúa a los estudiantes en las disciplinas de Lenguaje y Comunicación, Matemáticas, Ciencias Naturales, Historia, Geografía y Ciencias Sociales e Inglés, en los cursos de 20., 40. y 6o. y 8o. medio, con el fin de medir el logro de habilidades de aprendizajes (Ministerio de Educación de Chile [Mineduc])-, numerosos estudios concluyen en los bajos puntajes alcanzados por los estudiantes en dicha prueba (Bravo, Villalón y Orellana, 2004; Geegerat y Vásquez, 2008; Prieto y Contreras, 2008; Sotomayor, Parodi, Coloma, Ibáñez y Cavada, 2011). Todos se enfocan en el fracaso escolar y responsabilizan a los profesores de dicho fracaso. En los resultados, por ejemplo, en el caso de la asignatura de Lenguaje y Comunicación, se hace énfasis en la comprensión lectora, para lo cual Mineduc desarrolla propuestas que buscan fortalecer y apoyar el proceso de su enseñanza (Estándares de lectura 2o. y 4o. Básico, 2013; Consejo Nacional de la Cultura y Artes, 2010); sin embargo, los resultados siguen siendo insuficientes y los puntajes se mantienen bajos, situación que evidencia que las estrategias no han logrado una mejora significativa en los últimos años.

Esto último preocupa a los establecimientos escolares, sobre todo a escuelas municipales/públicas que son, en su mayoría, las que concentran los bajos puntajes (Zancajo, Bonal y Verger, 2015). Por esto, como mecanismo remedial, los establecimientos educativos han optado, primero, por realizar talleres SIMCE, y segundo, han centrado la atención en la cobertura curricular de los programas de estudios emanados desde el Mineduc. Estos antecedentes permiten afirmar que la evaluación de aprendizajes se entiende ahora en Chile como medición, y está centrada en evaluar sólo contenidos curriculares prescritos, con consecuencias más bien negativas, como la competencia entre establecimientos escolares, un ambiente de presión laboral docente y un proceso de evaluación homogéneo, provocando una brecha social, situación que estigmatiza a la escuela en función de puntajes y reduce la evaluación a un mero indicador de la calidad (Zancajo et al., 2015). En este punto cabe preguntarse si la naturaleza de los contenidos a evaluar determinados por el currículum nacional afecta los resultados que se han obtenido en contextos educativos vulnerables.

Estudios sobre el tema permiten reconocer posturas que van desde criticar la visión tecnocrática de los contenidos curriculares hasta investigaciones que amplían la gama de contenidos a enseñar y evaluar. Ferrada (2001) señala que los contenidos curriculares son importantes en cuanto valorizan la cultura racionalizada, pero con la finalidad de reconstruir una mentalidad globalizadora y holística, es decir, en tanto permitan a los estudiantes acceder a los conocimientos que se reconocen como válidos universalmente para un currículum prescrito. Flecha (1997, p. 33) plantea que el aprendizaje abarca todos los aspectos que se acuerda aprender, concluyendo así que los contenidos a enseñar no deben ser decididos "al margen de las personas, escudándose en razones de tipo técnico".

Sin embargo, existen dificultades en cuanto a la selección de contenidos no contemplados por el currículum prescrito, así lo evidencia Apple (1996):

Si bien todo el mundo tiene derecho, formalmente, a ser representado en los debates sobre la procedencia del capital cultural (saber "qué" conocimiento se declarará legítimo, "cómo" y "a quién") destinado a transmitirse a las futuras generaciones de estudiantes, ocurre que (...) aún sigue operando una tradición selectiva, en la que sólo se convierte en conocimiento oficial el conocimiento de determinados grupos. Así, la libertad para ayudar a seleccionar el corpus formal de conocimiento escolar está condicionada por las relaciones de poder, cuyos efectos son muy reales (p. 85). 
En el caso de Chile, estudios señalan la preeminencia de evaluación de contenidos curriculares sin considerar los contenidos de las culturas regionales del país, se excluye así a gran parte de la población indígena y persiste un "currículum homogéneo, estático, organizado y estructurado de manera que, si el profesorado de aula quisiera ampliarlos, debe homogenizarlos en función del planteamiento estatal" (Del Pino, Del Pino y Pincheira, 2016, p. 12).

No obstante, existen investigaciones que promueven la ampliación e incorporación de nuevos contenidos a la enseñanza escolar; Ferrer-Esteban (2007) da cuenta de la importancia de los contenidos curriculares, pero promueve que se considere la ampliación de éstos: "la organización de los contenidos de aprendizaje para que respondan a los criterios de diversidad y de acceso para todo el alumnado" (pp. 7071). En un estudio sobre profesores que se desempeñan en contextos de interculturalidad en Chile, Quilaqueo, Quintriqueo y Riquelme (2015) plantean que se deben incluir en los currículum de formación docente saberes culturales y de familias del pueblo mapuche, debido que el profesorado que se desempeña en dichos contextos genera un diálogo entre saberes escolares y mapuches. Estos contenidos deben ser incorporados en las escuelas para desplazar el currículum monocultural chileno, debiéndose aplicar una educación contextualizada que incluya los saberes indígenas, es decir, incorporar saberes propios de la familia y la comunidad mapuche (o indígena) al proceso de escolarización (Maheux, 2015; Quilaqueo, Quintriqueo y Riquelme, 2015).

Dichos estudios promueven el desarrollo curricular flexible, potenciando que en las escuelas se incorporen contenidos de tipo social, cultural, lingüístico e indígena, como respuesta a la diversidad cultural existente en el país, e indican que el proceso de evaluación de este tipo de contenidos no curriculares genera la igualdad de oportunidades de aprendizaje, sobre todo en estudiantes de contextos vulnerables.

Con base en estos antecedentes, un equipo de investigadores y profesores de universidades y escuelas ubicadas en la zona centro-sur de Chile, denominadas "Grupo enlazador de mundos", inició una línea de investigación enfocada en realizar una evaluación igualitaria, reflexiva y participativa, desde las voces de los protagonistas y con la convicción de que los principales factores que inciden en la evaluación del aprendizaje son: mejorar el ambiente en el aula, aligerar la presión por la medición, establecer redes de colaboración que permitan nivelar los aprendizajes y potenciar altas expectativas en el profesorado y estudiantes (Ferrada, 2008, 2012; Muskin, 2015). Dado lo anterior es que proponen el concepto de "Evaluación comunicativa" (Del Pino, 2013, 2014a, 2014b, 2015; Ferrada, 2008, 2012). El carácter "comunicativo" de la evaluación explica que las acciones evaluativas son coordinadas por el medio lingüístico, es decir, las personas involucradas en dichas acciones buscan alcanzar el entendimiento con base en argumentos susceptibles de crítica (Habermas, 1987).

El concepto de Evaluación comunicativa considera las voces de sus protagonistas para formular juicios de valor acerca de fenómenos importantes desde una óptica educativa, para un proceso de construcción de consensos sobre el programa educativo (Eisner, 1972; Guba y Lincoln, 1989; MacDonald, 1989; Parlett y Hamilton, 1989; Stake, 2006); se preocupa de aspectos de opresión y discriminación; la evaluación es un constructo de justicia social que reconoce las necesidades de la realidad (Mertens, 2013); y se comprende como un instrumento y práctica de mejora que fundamenta planes y proyectos de acción transformadora (Ferrer-Esteban, 2007).

La Evaluación comunicativa ha venido desarrollando en conjunto con el profesorado, estudiantes, familiares y demás colaboradores, algunos ejes que posibilitan su articulación en los establecimientos educativos (Del Pino, 2014a, 2015, 2016):

a) Finalidad de la evaluación. Cuando el profesorado incorpora a sus estudiantes e invita a las familias de ellos y otros voluntarios a participar en la toma de decisiones evaluativas, denominándose colaboradores de aprendizaje. Esto ocurre, por ejemplo, cuando estos agentes se reúnen a discutir los nudos problemáticos que interfieren en evaluación de aprendizajes y en conjunto delinean cómo abordar dichos nudos y quiénes participarán del proyecto de mejora evaluativa. De esta manera, su meta es común a toda la comunidad educativa, la cual se involucra solidariamente para mejorar distintas dimensiones de la evaluación, tales como notas, nivelación 
y rendimiento académico, entre otras.

b) Rol de los participantes. Cuando el profesorado invita a participar de las decisiones evaluativas a sus estudiantes y familias, rompe la jerarquía epistémica del rol docente como único conocedor del currículum e instala relaciones horizontales entre los agentes. Así, todos aportan desde sus saberes y experiencias en planos de igualdad para la superación de nudos problemáticos; esto ha evidenciado que cuando el docente trabaja con otros es posible atender a la totalidad de ritmos de aprendizaje de todo el estudiantado, ya que todos cumplen la función mediadora en la enseñanza y evaluación, posibilitando la apertura de tantos modos y formas de enseñanza y evaluación como personas trabajan en el aula.

c) Metodologías de evaluación: cuando todos los agentes involucrados en la mejora de las prácticas evaluativas, deciden cómo evaluar, surgen distintas estrategias. Entre las cuales se encuentran: grupos participativos de evaluación, que consiste en disponer el espacio de organización del aula con distintas actividades evaluativas que deben ser desarrollas en grupos por los estudiantes. Se promueve que los estudiantes roten por todas las actividades dispuestas. Esta estrategia puede desarrollarse en grupos de estudiantes y grupos conformados heterogéneamente por alumnos (género, estilos, rendimiento académico) y colaboradores de aprendizaje (edad, ocupación, grupo socioeconómico, etc.); círculos evaluativos, organización del aula en círculo o semicírculo que permite la evaluación del estudiante frente a sí mismo, la evaluación del estudiante frente a sus pares y la evaluación de todos los protagonistas; grupos comunicativos, organización en grupos dentro y fuera del aula, permite un trabajo interactivo que añade la corporeidad en actividades físicas como juegos, dramatizaciones, etc., junto a un trabajo teórico-práctico en aula; se evalúan habilidades cognitivas y de investigación en terreno (descubrimiento, síntesis, interpretación, etc.). Estas metodologías permiten que profesorado, familiares, voluntarios y estudiantes, desarrollen evaluaciones continuas formativas en todo el grupo, que trabajen colaborativamente en la construcción de distintas herramientas, ítems y actividades evaluativas, y que en conjunto sean capaces de identificar problemáticas puntuales y se centren en ellas (Del Pino, 2014a, 2014b, 2015).

Esta investigación busca responder a la siguiente interrogante: Considerando la naturaleza de los contenidos curriculares, ila evaluación comunicativa permite la integración de nuevos contenidos a evaluar? En estrecha relación con la pregunta, se formularon los siguientes objetivos: 1) Develar los contenidos que se trabajan, en evaluación comunicativa, con la perspectiva de todos los agentes educativos involucrados; 2) Identificar el rol de quienes participan en la selección de contenidos a evaluar, y 3) Identificar los criterios de selección de contenidos.

\section{Método}

El estudio se circunscribe al paradigma participativo (Gayá y Reason, 2009; Guba y Lincoln, 2012; Heron y Reason, 1997) con base en el enfoque dialógico-Kishu kimkelay ta che, originado desde la realidad chilena (Ferrada, 2010; Ferrada, Villena, Catriquir, Pozo, Turra, Schilling y Del Pino, 2014). El concepto dialógico explica que las problemáticas de investigación son construidas y desarrolladas con las propias personas y en sus propios contextos, a fin de producir procesos de transformación entre quienes participan (Ferrada, 1998, 2001). La frase mapuche Kishu kimkelay ta che significa "Ninguna persona conoce o aprende por sí misma" sino en conjunto con los demás en base a su legado histórico (Ferrada et al., 2014, p. 35). De esta forma, ambos conceptos coinciden en el carácter colectivo que tiene el conocimiento, comprensión que se configura en la visión epistémica de la investigación.

Con base a lo que propone la metodología empleada, la primera acción fue constituir una comunidad de investigación, que se fue organizando en el transcurso de tres años, del 2012 al 2015. Dicha comunidad estaba distribuida en cinco Instituciones educativas en Tomé, Concepción y Talca (estudiantes, profesores y colaboradores de aprendizaje), finalizando en una comunidad de 108 personas, incluido el investigador responsable (ver tabla I). Fue esta misma comunidad la que problematizó la pregunta de investigación, a fin proponer vías transformadoras para estudiantes de contextos educativos vulnerables. 
Tabla I. Comunidad de investigación

\begin{tabular}{|c|c|}
\hline \multicolumn{2}{|c|}{$\begin{array}{l}\text { Investigador Responsable: profesor y Doctor en Ciencias de la Educación, participante como comunidad de } \\
\text { investigación en todas las instituciones educativas. }\end{array}$} \\
\hline Institución & Participantes \\
\hline $\begin{array}{l}\text { Institución de Educación Básica y } \\
\text { Media, ubicada en sector urbano de } \\
\text { Tomé, con IVE de } 80 \%\end{array}$ & $\begin{array}{l}2 \text { profesores de Biología. } \\
4 \text { Colaboradores de aprendizaje, estudiantes de Pedagogía en Ciencias } \\
\text { con Mención Biología. } \\
23 \text { estudiantes de } 20 . \text { Medio. }\end{array}$ \\
\hline $\begin{array}{l}\text { Institución de Educación Básica y } \\
\text { Media, ubicada en sector urbano de } \\
\text { Concepción, con IVE de } 80.1 \%\end{array}$ & $\begin{array}{l}2 \text { profesores de Historia y Geografía. } \\
1 \text { Colaborador de aprendizaje estudiante de Ciencias Jurídicas. } \\
1 \text { Colaborador de aprendizaje estudiante de Enfermería. } \\
1 \text { Colaborador de aprendizaje estudiante de Pedagogía en Historia. } \\
26 \text { estudiantes de } 20 \text {. Medio. }\end{array}$ \\
\hline $\begin{array}{l}\text { Escuela de Educación Básica, } \\
\text { ubicada en zona rural de Talca, con } \\
\text { IVE de } 85 \%\end{array}$ & $\begin{array}{l}1 \text { profesor de Matemáticas. } \\
3 \text { estudiantes de Pedagogía General Básica. } \\
18 \text { estudiantes de } 50 \text {. Básico }\end{array}$ \\
\hline $\begin{array}{l}\text { Escuela de Educación Básica, } \\
\text { ubicada en zona rural de Talca, con } \\
\text { IVE de } 75 \%\end{array}$ & $\begin{array}{l}1 \text { profesor de Matemáticas. } \\
3 \text { estudiantes de Pedagogía General Básica. } \\
1 \text { asistente de aula. } \\
20 \text { estudiantes de } 60 \text {. Básico. }\end{array}$ \\
\hline
\end{tabular}

Las instituciones participantes presentan un alto Índice de Vulnerabilidad Educativa (IVE-SINAE), indicador del nivel de vulnerabilidad asociado a la "pobreza", como a la condición de riesgo asociada a los estudiantes de cada centro educativo. El índice empleado por el Sistema Nacional de Asignación con Equidad (SINAE) es una metodología de medición de la condición de vulnerabilidad que se construye con insumos de diferentes fuentes de información de cada estudiante (Encuestas de Vulnerabilidad de la Junta Nacional de Auxilio Escolar y Becas (JunAEB); Sistema de afiliación de Salud; Pertenecer a algún programa de la Red Sename (Servico Nacional de Menores); Pertenecer al Programa Chile Solidario o al Ingreso Ético Familiar; Información de Registro Civil y de Matrículas del Mineduc).

Las técnicas de recogida de información utilizadas fueron la conversación dialógica, que consta de un diálogo entre dos sujetos sobre un nudo problemático; los sujetos se pronuncian con sus demandas y argumentos con la finalidad de trasformar sus acciones para la mejora. Esta técnica se llevó a cabo en dos ocasiones con el profesorado, debido que poseen la experiencia de construcción y desarrollo curricular a diario, tomando decisiones curriculares y evaluativas frente a nudos problemáticos y demandas educativas. La otra técnica utilizada fue el diálogo colectivo, que consiste en un diálogo entre un conjunto de personas que frente a sus argumentos iniciales buscan la transformación, en función de acuerdos sobre la base de la mejora evaluativa. Se llevó a cabo en conjunto con colaboradores de aprendizaje y estudiantes; se realizaron dos diálogos colectivos por cada institución.

En cada una de las técnicas referidas se hizo un tratamiento de construcción de conocimiento propio de este tipo de investigación. En un primer momento la comunidad de investigación fue generando las categorías de estudio: Contenidos curriculares o no curriculares de evaluación, Selección de contenidos y Criterios de selección de contenidos (tabla II); dichas categorías se entienden como dispositivos genéricos que permiten definir y caracterizar los resultados en función de la transformación educativa que persiguen los sujetos. 
Tabla II. Categorías del estudio

\begin{tabular}{l|l}
\hline Categorías & Definición \\
\hline $\begin{array}{l}\text { Contenidos } \\
\text { curriculares y } \\
\text { no curriculares }\end{array}$ & $\begin{array}{l}\text { - Se entiende por contenido curricular (o instrumental) aquel conocimiento establecido por el } \\
\text { - Se entiende por contenido no curricular aquel conocimiento o saber no sistematizado para su } \\
\text { enseñanza y evaluación en la escuela, que corresponde a conocimientos propios de la cultura } \\
\text { social, folklórica, lingüística y religiosa que los sujetos y comunidades portan. } \\
\text { - Se entiende por selección de contenidos aquellos acuerdos alcanzados por los sujetos sobre } \\
\text { qué contenidos (curriculares o no curriculares, o ambos) evaluar, que potencien sentido } \\
\text { pedagógico a los estudiantes. }\end{array}$ \\
$\begin{array}{l}\text { Criterios de } \\
\text { selección }\end{array}$ & $\begin{array}{l}\text { - Se entiende por criterios de selección aquellos principios, normas o valoraciones que tienen } \\
\text { relación con un juicio valorativo sobre el objeto a evaluar. }\end{array}$ \\
\hline
\end{tabular}

Fuente: Parcialmente extraída de Del Pino (2014a).

Un segundo momento consistió en que cada técnica fue transcrita, elaborándose una base de datos a la que se aplicó una técnica para determinar su carácter comunicativo o no (Ferrada, 2010). En un tercer momento se categorizó el conocimiento construido sobre la base de la distinción de dimensiones transformadoras, conservadoras y exclusoras. La dimensión transformadora se entienden como conocimiento de evaluación construido colectivamente -identificado como aspectos que contribuyen al proceso que se persigue transformar; la dimensión conservadora es el conocimiento construido que puede ser identificado como aquellos aspectos que obstaculizan el proceso transformador que se persigue; y la exclusora es aquel conocimiento construido que es identificado como aspectos que deben ser conservados. Por último, se organizó el conocimiento acordado como definitivo en carácter transformador y conservador, y el de carácter exclusor sirvió de base para investigaciones que ahora se encuentran en desarrollo.

\section{Resultados}

En relación a la pregunta de si la evaluación comunicativa permite la integración de nuevos contenidos a evaluar, los resultados se presentan en función de cada objetivo en las tablas III, IV y V, identificándose la categoría, subcategoría, incidentes extraídos de los registros de las técnicas de recogida de datos a modo de ilustración y la dimensión en que fueron validados: Transformadores (Tr), Conservadores (C) y Exclusores (Ex). 
Tabla III. Resultados de la categoría Contenidos

\begin{tabular}{|c|c|c|c|c|}
\hline \multirow{2}{*}{ Subcategoría } & \multirow{2}{*}{ Extractos de registros de datos } & \multicolumn{3}{|c|}{ Dimensiones } \\
\hline & & $\mathrm{Tr}$ & C & Ex \\
\hline $\begin{array}{l}\text { Contenidos } \\
\text { curriculares como } \\
\text { fundamentales }\end{array}$ & $\begin{array}{l}\text { "el contenido [curricular] ... es importante, porque es fundamental, es lo } \\
\text { abstracto, y ellos [estudiantes] pueden relacionarlo en su casa, por } \\
\text { ejemplo el cáncer, con factores como el cigarro..." (Ciudad de Tomé, } \\
\text { 2012, Diálogo Colectivo, P5) }\end{array}$ & & & $x$ \\
\hline $\begin{array}{l}\text { Contenidos } \\
\text { curriculares } \\
\text { contextualizados }\end{array}$ & $\begin{array}{l}\text { "hay que contextualizar [los contenidos] en el sentido que se está } \\
\text { trabajando en un contexto de vulnerabilidad [...] en esta perspectiva } \\
\text { evaluativa se practica el hecho de buscar una sociedad dialógica..." } \\
\text { (Ciudad de Concepción, 2013, Diálogo Colectivo, P4) }\end{array}$ & & $x$ & \\
\hline $\begin{array}{l}\text { Contenidos } \\
\text { socioculturales y } \\
\text { curriculares }\end{array}$ & $\begin{array}{l}\text { "el contenido vivencial es el que le da sentido a los contenidos del } \\
\text { currículum, porque los asocian a su experiencia directa. [...] si tú les vas a } \\
\text { hablar de trabajo, muchos de los que están allí saben lo que es trabajar, } \\
\text { entonces tú no tienes que explicarles lo que es la desocupación, lo que } \\
\text { es la cesantía, porque si no lo han vivido ellos, lo han vivido sus familias." } \\
\text { (Ciudad de Concepción, 2013, Diálogo Colectivo, P2). }\end{array}$ & $x$ & & \\
\hline $\begin{array}{l}\text { Contenidos desde } \\
\text { la promoción de } \\
\text { distintas } \\
\text { habilidades }\end{array}$ & $\begin{array}{l}\text { "ha sido más fácil aprender, porque nos evalúan así como con juegos } \\
\text { matemáticos o con contenidos de lenguaje para aprender matemáticas y } \\
\text { aprendemos más de las clases y las evaluaciones [...] yo, por ejemplo, } \\
\text { evalué a mis compañeros haciendo fracciones con su cuerpo... [...] lo } \\
\text { que se enseña y evalúa potencia muchas habilidades..." (Ciudad de Talca, } \\
\text { 2015, Diálogo Colectivo, P9, } 11 \text { y 12). }\end{array}$ & $x$ & & \\
\hline $\begin{array}{l}\text { Contenidos } \\
\text { lingüísticos de su } \\
\text { contexto cultural }\end{array}$ & $\begin{array}{l}\text { "Un lenguaje propio de su cultura, o sea, su familia, y lo otro, que los } \\
\text { niños finalmente usan ese lenguaje como contenido porque es propio de } \\
\text { su cultura y les favorece en la escuela". (Ciudad de Talca, 2015, Diálogo } \\
\text { Colectivo, P2). }\end{array}$ & $x$ & & \\
\hline
\end{tabular}

Se observa que dentro de los resultados obtenidos hay perspectivas disímiles acerca de los contenidos que se evalúan cuando se trabaja con la evaluación comunicativa. Por un lado se consideran importantes los contenidos curriculares, y por otro - desde la dimensión conservadora y transformadora, teniendo como base lo anterior- se promueve una ampliación de contenidos no considerados en el currículum, para dotar de "contexto situado" a los conocimientos y desarrollar una evaluación dirigida a valorar distintos tipos de habilidades.

Tabla IV. Resultados de la categoría Selección de contenidos

\begin{tabular}{|c|c|c|c|c|}
\hline \multirow{2}{*}{ Subcategoría } & \multirow{2}{*}{ Extractos de registros de datos } & \multicolumn{3}{|c|}{ Dimensiones } \\
\hline & & $\mathrm{Tr}$ & $\mathrm{C}$ & Ex \\
\hline $\begin{array}{l}\text { Solamente los } \\
\text { profesores } \\
\text { seleccionan los } \\
\text { contenidos a } \\
\text { evaluar }\end{array}$ & $\begin{array}{l}\text { "yo creo que desde la perspectiva de nosotros como profesores es más } \\
\text { difícil adecuarnos a este tipo de evaluación, a eso voy, nosotros creo que } \\
\text { debemos seleccionar los contenidos que se evalúan..." (Ciudad de Tomé, } \\
\text { 2012, Conversación Dialógica, P6) }\end{array}$ & & & $x$ \\
\hline $\begin{array}{l}\text { Todas las } \\
\text { personas } \\
\text { participan de la } \\
\text { selección de } \\
\text { contenidos }\end{array}$ & $\begin{array}{l}\text { "[la selección de contenidos] debería ser no sólo las personas que estamos } \\
\text { haciendo las clases, sino con la colaboración de todos los que estamos } \\
\text { participando dentro del proyecto [...] de esa manera podríamos abarcar } \\
\text { aquellas cosas que nosotros no tenemos pensado o estipulado, personas } \\
\text { que capaz estén inmersos en la clase nos puedan decir que esto sí es } \\
\text { necesario evaluar [...] todos deben ser partícipes para poder consensuar } \\
\text { qué tipo de contenidos vamos a evaluar..." (Ciudad de Tomé, 2012, } \\
\text { Conversación Dialógica, RC 3). }\end{array}$ & $x$ & & \\
\hline
\end{tabular}

Fuente: elaboración propia.

Se observa en los resultados dos tipos de roles: por un lado, la negación docente que otros puedan aportar a la selección de contenidos y su evaluación genera una postura tradicional del profesor como único responsable de la enseñanza de los estudiantes; por otro lado, la dimensión transformadora explica 
que cuando se trabaja con la evaluación comunicativa los participantes promueven establecer planos horizontales de trabajo colaborativo para la selección de contenidos de evaluación.

Tabla V. Resultados de la categoría Criterios de selección

\begin{tabular}{|c|c|c|c|c|}
\hline \multirow{2}{*}{ Subcategoría } & \multirow{2}{*}{ Extractos de registros de datos } & \multicolumn{3}{|c|}{ Dimensiones } \\
\hline & & $\mathrm{Tr}$ & C & Ex \\
\hline $\begin{array}{l}\text { Criterio de un } \\
\text { experto }\end{array}$ & $\begin{array}{l}\text { "entonces la edad es un factor para la selección, el criterio de selección de } \\
\text { contenidos tendría que ser por un experto, porque ellos [los estudiantes] va } \\
\text { en relación a su edad... [...] un experto sabe qué contenidos deben evaluarse, } \\
\text { por lo tanto, el criterio es su especialidad, su experiencia en esto como un } \\
\text { experto..." (Ciudad de Tomé, 2012, Diálogo Colectivo, P7, P8) }\end{array}$ & & & $x$ \\
\hline $\begin{array}{l}\text { Coordinación } \\
\text { para alcanzar el } \\
\text { entendimiento }\end{array}$ & $\begin{array}{l}\text { "lo que se pretende evaluar la persona [profesores, estudiantes y } \\
\text { colaboradores de aprendizaje] participa en la construcción de la evaluación y } \\
\text { en los criterios de selección, eso implica, que la persona vuelve sobre el } \\
\text { contenido, respeta ese principio de que no se puede evaluar de lo que no se } \\
\text { ha visto, no se ha trabajado [...] entonces, por eso es importante } \\
\text { coordinarnos para seleccionar los contenidos en conjunto..." (Ciudad de } \\
\text { Concepción, 2013, Diálogo Colectivo, P3) }\end{array}$ & $x$ & & \\
\hline $\begin{array}{l}\text { Creación de } \\
\text { sentido }\end{array}$ & $\begin{array}{l}\text { "a los estudiantes no les vas a hablar de pobreza, porque ellos conocen la } \\
\text { pobreza, tampoco cómo se les puede evaluar esos contenidos, lo importante } \\
\text { aquí es que uno de los criterios debe velar por la creación de sentido de los } \\
\text { contenidos [...] la creación de sentido quiere decir que los contenidos les } \\
\text { abran las puertas a otros conocimientos que les permitan [a los estudiantes] } \\
\text { desear superar su condición de pobreza" (Ciudad de Concepción, 2013, } \\
\text { Conversación Dialógica, P1). }\end{array}$ & $x$ & & \\
\hline $\begin{array}{l}\text { Autonomía en la } \\
\text { toma de decisión }\end{array}$ & $\begin{array}{l}\text { "se trabaja en un plano de igualdad, todos aportamos de la misma manera y } \\
\text { eso es un criterio para seleccionar contenidos, que profesores, colaboradores } \\
\text { y estudiantes hemos sido autónomos, por darte un ejemplo, los chicos han } \\
\text { propuesto contenidos y cómo quieren ser evaluados, han propuesto } \\
\text { verdaderos y falso, juegos, selección múltiple, pero la propuesta viene de } \\
\text { ellos [...] todo se hace en función de un consenso, pero ese consenso es } \\
\text { gracias a la autonomía que funciona como criterio de selección de contenidos } \\
\text { en este tipo de evaluación comunicativa..." (Ciudad de Concepción, 2013, } \\
\text { Conversación Dialógica, P5). }\end{array}$ & $x$ & & \\
\hline
\end{tabular}

Fuente: elaboración propia.

Se observa que la mayoría de criterios identificados rescatan y promueven la participación de todos los sujetos implicados en el trabajo de evaluación, posibilitando criterios de selección que contribuyan a los consensos por medio de argumentos, toma de decisión autónoma y que los contenidos seleccionados tengan la particularidad de abrir horizontes de conocimientos nuevos con base a los contenidos curriculares.

\section{Discusión y conclusiones}

La investigación revela, con relación al objetivo 1, que profesorado, estudiantes y colaboradores de aprendizaje sostienen la necesidad de ampliar los contenidos a evaluar, no en desmedro de los contenidos curriculares/instrumentales, sino enriqueciéndolos con y desde saberes socioculturales, lingüísticos u otros, debido que representan la historia viva en la estructura social.

(...) podría hablarte del tema de los indígenas, hay muchos jóvenes que ven y sienten que aún hay muchos indígenas que se les margina, entonces comprender la realidad y la importancia de estos contenidos, por ejemplo, los indígenas en la conformación de nuestra sociedad... (Ciudad de Concepción, 2013, Diálogo Colectivo, P2).

La cita confirma que los contenidos que no están considerados en el currículum ministerial podrían posibilitar mayores expectativas de comprensión de problemáticas sociales en los estudiantes y el proyecto evaluador se puede concentrar en generar vías de acceso a soluciones. Retomando a Apple 
(1996), la superación de contenidos curriculares es de carácter epistémico, debido que cada contenido evaluado es una construcción de realidad que proporciona una creación y apertura de creación de sentidos de vida y proyectos comunes sobre la mejora educativa. Estos resultados responden a características históricas de los contenidos de la evaluación. De acuercon con Ferrada (2001), los contenidos curriculares son parte de una cultura racionalizada, y esto motiva a la comunidad de investigación a rescatar y proponer la evaluación de contenidos socioculturales que guardan relación con las costumbres y el folklore, entre otros, generando una propuesta que valida un rescate de la historicidad de los conocimientos como organización de contenidos evaluativos. Esto se observa, por ejemplo, cuando un colaborador de aprendizaje señala:

(...) el contenido vivencial es el que le da sentido a los contenidos del currículum, porque los asocian a su experiencia directa. [...] si tú les vas a hablar de trabajo, muchos de los que están allí saben lo que es trabajar, entonces tú no tienes que explicarles lo que es la desocupación, lo que es la cesantía, porque si no lo han vivido ellos, lo han vivido sus familias (Ciudad de Concepción, 2013, Diálogo Colectivo, P2).

Los contenidos que se proponen evaluar deben potenciar diversas habilidades, es decir, un contenido cultural, como el aprendizaje de una lengua indígena, que desarrolla la habilidad lingüística de los estudiantes y amplía a la vez la concepción de realidad, no circunscrita al conocimiento occidentalizado. Dice Maheux (2015, p. 43) que "la cuestión de los idiomas en contacto, es el centro de los procesos de escolarización de los estudiantes". Lo que se vincula directamente con contenidos lingüísticos de contextos culturales, debido que el aprendizaje de la lengua materna y de otras lenguas posibilita el ingreso a una cultura tanto occidentalizada como no, es decir, aspectos que las comunidades indígenas y otras comunidades latinoamericanas todavía mantienen (aspectos religiosos, ceremoniales, socioculturales, etc.).

En relación con lo anterior, los resultados del objetivo 2 y 3 promueven una selección de contenidos participativa y que los criterios de selección amplíen el horizonte de contenidos para los estudiantes y no los circunscriban a un tipo de conocimiento monocultural. Esto tiene relación con lo dicho por Zemelman (2005), quien señala que la realidad es histórico-social con potencialidad de futuro, donde el incluir a los sujetos en la construcción de conocimiento implica "transformar la externalidad de objeto en un ámbito de sentidos susceptibles de ser potenciados desde diversas opciones de futuro" (p. 92). El profesorado, estudiantes y colaboradores recuperan conocimientos (traducidos en contenidos) que han sido desplazados por el consumo mercantil de la educación. Plantean Apple (1996) y Ferrer-Esteban (2007), que son aquellos saberes no mencionados por la cultura oficial los que permiten conocer la realidad y dotar de sentido a la evaluación como una herramienta de potencial transformador.

Lo anterior tiene correspondencia con el planteamiento de Del Pino, Del Pino y Pincheira (2016): el curriculum nacional es estático, por lo tanto, es impuesto a los docentes, quienes no tienen incidencia en la selección de contenidos a enseñar y evaluar. Esta investigación contribuye a estudios similares (Del Pino, Del Pino y Pincheira, 2016; Maheux, 2015; Quilaqueo, Quintriqueo y Riquelme, 2015), en cuanto a que caracteriza conceptualmente qué contenidos no considerados en el currículum oficial podrían incidir en la mejora educativa de establecimientos caracterizados como vulnerables; sumándose a las investigaciones anteriores que validan el trabajo de evaluación comunicativa, la promoción de transformación educativa por medio de contenidos, tanto curriculares como socioculturales, dando el carácter de temporalidad a los mismos.

Estos contenidos representan la historia viva, articulando el tiempo en un presente contextual. Por eso no se puede sólo evaluar contenidos disciplinares, debido que no representan el tiempo presente y no permiten su articulación con la realidad, por el contrario, este componente transformativo lo dan los contenidos propios de las culturas contextuales. De esta manera, la evaluación comunicativa permite a la comunidad de investigación reconocer el componente gnoseológico de la evaluación; para Freire (2007) lo gnoseológico cumple una función de historización, promueve la reflexión del hombre con el mundo, a tener conciencia de él y a nombrar al mundo en la palabra en acciones solidarias y comunitarias. Por ello, la evaluación es un constructo epistemológico que construye conocimiento educativo tanto para develar demandas y nudos problemáticos evaluativos, como para propiciar vías de articulación para la 
transformación hacia la mejora.

La integración de contenidos desde la evaluación comunicativa permite entender a las personas como seres capaces de intervenir en el mundo por medio de la palabra, por ello se constituye desde los componentes experienciales y prácticos, porque representa las demandas evaluativas de una determinada realidad educativa, y se constituye como una herramienta de acompañamiento y transformación hacia la mejora. Estos contenidos que se integran al proceso de aprendizaje y evaluación reconocen y comprenden una diversidad de saberes que portan los sujetos y que posibilitan una mayor creación de sentido de vida a estudiantes de contextos educativos de alta vulnerabilidad, lo que permite construir proyectos de evaluación educativa que ayuden superar el fracaso escolar.

\section{Referencias}

Apple, M. (1996). El conocimiento oficial. La educación democrática en una era conservadora. Madrid: Paidós.

Bravo, L., Villalón, M. y Orellana, E. (2004). Los procesos cognitivos y el aprendizaje de la lectura inicial: diferencias cognitivas entre buenos lectores y lectores deficientes. Revista Estudios Pedagógicos, 30, 7-19. Recuperado de http://revistas.uach.cl/index.php/estped/article/view/3112

Consejo Nacional de la Cultura y las Artes. (2010). Estudio sobre el comportamiento lector a nivel nacional. Santiago de Chile: Gobierno de Chile. Recuperado de http://plandelectura.gob.cl/recursos/estudio-sobrecomportamiento-lector-a-nivel-nacional-2011/

Del Pino, M. (2013). Competencias iniciales en la formación de profesores en evaluación dialógicacomunicativa. En D. Ferrada y R. Ruay (Comps.), Práctica educativa y social en la universidad, escuela y comunidad (pp. 305-317). Chile: Universidad Universidad de Concepción. Recuperado de

https://www.researchgate.net/publication/308379149 Competencias iniciales de formacion de profes ores en evaluacion dialogico-comunicativa

Del Pino, M. (2014a). Evaluación comunicativa: un aporte desde el modelo dialógico de la pedagogía Enlazando Mundos. Tesis Doctoral. Facultad de Filosofía y Letras. Universidad Nacional de Cuyo, Argentina. Recuperado de http://bdigital.uncu.edu.ar/objetos digitales/11210/morchioydifabio-tesisdoctorales cienciaseducacionffyl.pdf

Del Pino, M. (2014b). Caracterización teórica del modelo de evaluación comunicativa. Revista de Evaluación Educativa, 3(1). Recuperado de

https://www.researchgate.net/publication/308334447 Caracterizacion teorica del modelo de evaluaci on comunicativa

Del Pino, M. (2015). Sistematización de la experiencia de evaluación comunicativa (2011-2014). Revista Electrónica Diálogos Educativos, 15(29), 106-123. Recuperado de http://revistas.umce.cl/index.php/dialogoseducativos/article/view/1022

Del Pino, M. (2016). Comunidad de evaluación: protagonistas en las decisiones. Revista EDUCERE, 20(65), 61-71. Recuperado de http://www.saber.ula.ve/handle/123456789/42217

Del Pino, M., Del Pino, A. y Pincheira, D. (2016). La lectura desde el enfoque del Ministerio de Educación y el enfoque dialógico de la pedagogía Enlazando Mundos. Revista Electrónica Educare, 20(3), 1-21.

Eisner, E. W. (1998/1972). Educar la visión artística. España: Paidós.

Ferrada, D. (1998). El currículum crítico comunicativo y la selección de la cultura escolar. Tesis Doctoral. Universidad de Valladolid, España. 
Ferrada, D. (2001). Currículum crítico comunicativo. Barcelona: El Roure.

Ferrada, D. (2008). Enlazando mundos: un modelo pedagógico que construye esperanzas de igualdad e inclusión en escuelas públicas. Revista Rexe, 7(14), 37-52. Recuperado de http://www.rexe.cl/14/143.htm

Ferrada, D. (2010). Tratamiento de la construcción del conocimiento en la investigación dialógica-Kishu Kimkelay Ta Che. Documento de trabajo Grupo Enlazador de Mundos, no publicado.

Ferrada, D. (2012). Construyendo escuela, compartiendo esperanzas. Chile: RiL editores.

Ferrada, D., Villena, A., Catriquir, D., Pozo, G., Turra, O., Schilling, C. y Del Pino, M. (2014). Investigación dialógica-Kishu Kimkelay Ta Che en educación. Revista Rexe, 13(26) 33-50. Recuperado de http://www.rexe.cl/ojournal/index.php/rexe/article/view/32

Ferrer-Esteban, G. (2007). Las comunidades de aprendizaje y su sostenibilidad. Hacia un modelo de evaluación inclusiva. En J. Majado, J. Rodríguez, J. Vera, M. Zafra, M. Castro, M. H. Zapico y G. FerrerEsteban. La escuela en la comunidad. La comunidad en la escuela (pp. 39-78). España: Graó.

Flecha, R. (1997). Compartiendo palabras: el aprendizaje de las personas adultas a través del diálogo. España: Paidós.

Freire, P. (2007). Pedagogía del oprimido. Madrid: Siglo XXI editores.

Gayá, P. y Reason, P. (2009). Initiating action research: Challenges and paradoxes of opening communicative space. Action Research, 7(3), 243-262. doi:10.1177/1476750309336715

Geeregat, O. y Vásquez, O. (2008). Crisis y temporalidad en la formación inicial de profesores de lenguaje y comunicación. Revista Estudios Pedagógicos 34(2) 87-98.

Guba, E. G. y Lincoln, Y. S. (1989). Fourth generation evaluation. EUA: Sage.

Guba, E. y Lincoln, Y. (2012). Controversias paradigmáticas, contradicciones y confluencias emergentes. En N. Denzin e Y. Lincoln (Comps.). Manual de investigación cualitativa (pp. 38-78) Argentina: Gedisa.

Habermas, J. (1987). Teoría de la acción comunicativa: racionalidad de la acción y racionalización social (vol. 1). Madrid: Taurus.

Heron, J. y Reason, P. (1997). A Participatory Inquiry Paradigm. Qualitative Inquiry, 3(3), 274-294. doi:10.1177/107780049700300302

MacDonald, B. (1989). La evaluación y el control de la educación. En J. G. Sacristán y A. Pérez (Eds.), La enseñanza: su teoría y su práctica (pp. 467-478). Madrid: Akal.

Maheux, G. (2015). Formación del profesorado Inuit y de las primeras Naciones en Québec: Problemática y pistas de acción. En D. Quilaqueo, S. Quintriqueo y F. Peña (Ed.). Interculturalidad en contexto de diversidad social y cultural (pp. 35-51) Chile: Ediciones Universidad Católica de Temuco.

Mertens, D. (2013). Social transformation and evaluation. En M. Alkin (Ed.), Evaluation roots (pp. 229-240). EUA: Sage. 
Muskin, J. A. (2015). Evaluación del aprendizaje del estudiante y el currículo: problemas y consecuencias para la política, el diseño y la aplicación. Reflexiones en Progreso, 1, 1-32. Recuperado de http://www.ibe.unesco.org/es/documento/evaluaci\%C3\%B3n-del-aprendizaje-del-estudiante-y-elcurr\%C3\%ADculo-problemas-y-consecuencias-para-la

Parlett, M. y Hamilton, D. (1989). La evaluación como iluminación. En J. Gimeno y A. Pérez (Eds.), La enseñanza: su teoría y su práctica (pp.450-466). Madrid, España: Akal.

Prieto, M. y Contreras, G. (2008). Las concepciones que orientan las prácticas evaluativas de los profesores: un problema a develar. Revista Estudios Pedagógicos, 34 (2), 245-262.

Quilaqueo, D., Quintriqueo, S. y Riquelme, E. (2015). Identidad profesional docente: profesores de pedagogía en educación básica intercultural en contexto mapuche. En D. Quilaqueo, S. Quintriqueo y F. Peña (Eds.), Interculturalidad en contexto de diversidad social y cultural (pp. 237-262). Chile: Universidad Católica de Temuco.

Sotomayor, C., Parodi, G., Coloma, C., Ibáñez, R. y Cavada, P. (2011). La formación inicial de docentes de Educación General Básica en Chile. ¿Qué se espera que aprendan los futuros profesores en el área de Lenguaje y Comunicación? Pensamiento Educativo. Revista de Investigación Educacional Latinoamericana, 48(1), 28-41. Recuperado de http://pensamientoeducativo.uc.cl/index.php/pel/article/view/446/1692

Stake, R. E. (2006). Evaluación comprensiva y evaluación basada en estándares. España: Graó.

Zancajo, A., Bonal, X. y Verger, A (2015). Mercados educativos y segmentación de la oferta escolar: efectos sobre las desigualdades educativas en Chile. En V. Vidal (Org). Diálogos sobre as redefinições no papel do Estado e nas fronteiras entre lo publico e o privado na educação (pp. 216-236). Brasil: Oikos.

Zemelman, H. (2005). Voluntad de conocer. El sujeto y su pensamiento en el paradigma crítico. España: Anthropos. 